\title{
Knuckle pads-leukonychia-sensorineural deafness-palmoplantar hyperkeratosis syndrome
}

INSERM

\section{Source}

INSERM. (1999). Orphanet: an online rare disease and orphan drug data base. Knuckle pads-leukonychia-sensorineural deafness-palmoplantar hyperkeratosis syndrome. ORPHA:2698

A rare, syndromic genetic deafness disease characterized by symmetric or asymmetirc knuckle pads (typically located on the distal and interphalangeal joints), leukonychia, diffuse palmoplantar keratoderma, and congenital, mild to moderate sensorineural deafness. 